\title{
Block method with Hermite interpolation for numerical solution of delay differential equations
}

\begin{abstract}
In this paper, we consider a two-point implicit block method for solving delay differential equations. For greater efficiency, the block method is implemented in variable stepsize technique. The most optimal stepsize is taken while achieving the desired accuracy. The implicit method is solved using predictor-corrector scheme where the corrector is iterated until convergence. Grid point formulae are derived using a predictor and a corrector of order five. The formulae produce two new values in a single integration step. Delay solutions are approximated using Hermite interpolation of order five. The advantage of using Hermite interpolation is that it requires less support points than the existing interpolation technique in order to achieve the overall accuracy. Numerical results indicate that the two-point block method with Hermite interpolation technique is efficient and reliable in solving a wide range of delay differential equations.
\end{abstract}

Keyword: Block method; Delay differential equations; Hermite interpolation; Numerical solution 